\title{
Development and evaluation of a tutorial to improve students' understanding of a lock-in amplifier
}

\author{
Seth DeVore, Alexandre Gauthier, Jeremy Levy, and Chandralekha Singh \\ Department of Physics and Astronomy, University of Pittsburgh, Pittsburgh, Pennsylvania 15260, USA
}

(Received 12 October 2015; published 6 September 2016)

\begin{abstract}
A lock-in amplifier is a versatile instrument frequently used in physics research. However, many students struggle with the basic operating principles of a lock-in amplifier which can lead to a variety of difficulties. To improve students' understanding, we have been developing and evaluating a research-based tutorial which makes use of a computer simulation of a lock-in amplifier. The tutorial is based on a field-tested approach in which students realize their difficulties after predicting the outcome of simulated experiments involving a lock-in amplifier and check their predictions using the simulated lock-in amplifier. Then, the tutorial provides guidance and strives to help students develop a coherent understanding of the basics of a lock-in amplifier. The tutorial development involved interviews with physics faculty members and graduate students and iteration of many versions of the tutorial with professors and graduate students. The student difficulties with lock-in amplifiers and the development and evaluation of the research-based tutorial to help students develop a functional understanding of this device are discussed.
\end{abstract}

DOI: 10.1103/PhysRevPhysEducRes.12.020127

\section{INTRODUCTION}

The physics graduate student population is one of the most under-addressed groups in physics education research (PER). While the group represents students with many years of directed study within the field of physics, it is difficult to find research-based tools with the express intent of addressing student difficulties of physics graduate students and providing scaffolding support for the continued education of those at the highest tiers of physics learning. However, not only can PER help physics graduate students develop functional understanding of physics in the graduate level courses, PER-based tools can also be helpful for them once they move beyond the classroom and into their roles as researchers. While these students have been through the entire undergraduate physics curriculum, they can still benefit from research-based intervention to help them learn, e.g., with tutorials based upon physics education research.

To aid in physics graduate students' entry into the lab setting that requires the use of a lock-in amplifier, we developed and evaluated a research-based tutorial to help students develop a functional understanding of this device. The tutorial strives to help students integrate conceptual and quantitative aspects of how this device works so that they can use the device effectively and troubleshoot problems that may arise.

Published by the American Physical Society under the terms of the Creative Commons Attribution 3.0 License. Further distribution of this work must maintain attribution to the author(s) and the published article's title, journal citation, and DOI.
The lock-in amplifier (lock-in) is an instrument used extensively in lab research, especially in condensed matter physics [1-6]. The most common use of a lock-in in laboratory research is to measure small signals that are synchronous with an external reference, often in the presence of large background signals (or "noise"). However, many students who use this instrument for their research have only a limited understanding of the operation of lock-ins. Often, the lock-in is used as a "black box" to find the amplitude of a signal at a given frequency without understanding the instrument's internal workings. According to the experimental condensed matter physics faculty members interviewed by us during this researchproject, improper or inefficient use of the lock-in and misinterpretation of data obtained from the device are quite common among the students in their research lab. Additionally, according to these faculty advisors, the lack of understanding can often result in students being unable to troubleshoot and modify the experimental setup when faced with anomalous results.

Computer-based and web-based learning tools are becoming increasingly common to aid in learning across many science and engineering fields [7-18]. These tools must be developed using a research-based approach to ensure that they are effective and suit both the level and prior experience of the students they are intended for $[19,20]$. Here, we discuss the development and evaluation of the lock-in tutorial, which makes use of a simulation of the lock-in device to ease the transition of those who are just beginning to use this instrument in their research in the lab setting, as well as to provide a firmer foundation for those who already use lock-ins in their research. Our goal in developing this research-based tutorial was to help students 
understand the basics of the lock-in functions. In particular, the tutorial strives to help students who use (or plan to use) lock-ins in their research understand more deeply how the input parameters in diverse situations affect the output and why, so that they learn to predict the output of the device for a set of input parameters. In particular, by merging conceptual and mathematical aspects of the operation of the instrument, the tutorial strives to help students learn the relationship between the input parameters and expected outputs so that they are able to troubleshoot unexpected outputs in their lab work if the output does not seem consistent with the input they used (e.g., due to various types of noise or inappropriate setting of the time constant for a given experiment).

In the following sections, we will begin with a description of the idealized lock-in in which we will summarize the mathematical preliminaries required, because integrating conceptual and quantitative aspects of the device is critical for a robust understanding of the device. This section is followed by the methodology used in the development of the tutorial, including methods for investigating student difficulties in using this device. We then summarize the structure of the tutorial, including the pretest and post-test and the associated simulation. This is followed by an examination of the student difficulties that came to light in our initial investigation and emerged from think-aloud interviews, in which students made use of early versions of the tutorial. Investigation of these student difficulties was critical for addressing them using a guided approach to learning, used in the lock-in tutorial. Finally, we discuss the evaluation of the tutorial using a pretest and post-test. While a brief article about this tutorial was recently published elsewhere [21], this paper will go into substantial additional detail regarding the process by which the tutorial was developed and evaluated, as well as the common difficulties faced by students making use of the lock-in.

\section{THE IDEAL LOCK-IN AMPLIFIER}

Throughout this paper, as in the tutorial, we will treat the lock-in as an idealized version of the instrument. Here we assume that the signal of interest is centered on a frequency $f_{S}$ which is present in the input signal. In general, it will not be a pure frequency since the amplitude can change, and amplitude modulation leads to sidebands that surround the central frequency. In the case in which there is no amplitude modulation introduced into the signal, we will treat this frequency as a pure frequency. In this tutorial, we make use of both idealized pure frequencies and input signals that are undergoing amplitude modulation to help students learn about both treatments of the lock-in, though in this section we will strictly concern ourselves with pure frequencies. To separate the signal of interest from unwanted noise, a reference signal is defined. The reference signal is selected to have a unit amplitude (for convenience). The (idealized) single-frequency input signal is first preamplified by a factor $g$ to give

$$
V_{I}=g A_{S} \cos \left(2 \pi f_{S} t+\varphi\right) .
$$

This amplified signal is then multiplied (or "mixed") by a reference for the $x$ and $y$ channels of the lock-in:

$$
\begin{aligned}
& v_{R X}=\cos \left(2 \pi f_{R} t\right), \\
& v_{R Y}=-\sin \left(2 \pi f_{R} t\right),
\end{aligned}
$$

to form the "unfiltered" $x$ - and $y$-channel outputs of the lock-in:

$$
\begin{aligned}
& V_{M X}(t)=V_{I}(t) v_{R X}(t), \\
& V_{M Y}(t)=V_{I}(t) v_{R Y}(t) .
\end{aligned}
$$

Here, $\varphi$ is the phase of the input signal of frequency $f_{S}$ with respect to the reference signal, and $A_{S}$ is the amplitude of the input signal with frequency $f_{S}$. To understand the effect of the mixer, we rely on two trigonometric identities:

$$
\begin{aligned}
& \cos (a) \cos (b)=1 / 2[\cos (a+b)+\cos (a-b)], \\
& \cos (a) \sin (b)=1 / 2[\sin (a+b)-\sin (a-b)] .
\end{aligned}
$$

Application of these identities yields

$$
\begin{aligned}
V_{M X}= & V_{I} v_{R X}=\frac{1}{2} g A_{S}\left\{\cos \left[2 \pi\left(f_{S}-f_{R}\right) t+\varphi\right]\right. \\
& \left.+\cos \left[2 \pi\left(f_{S}+f_{R}\right) t+\varphi\right]\right\}, \\
V_{M Y}= & V_{I} v_{R Y}=\frac{1}{2} g A_{S}\left\{\sin \left[2 \pi\left(f_{S}-f_{R}\right) t+\varphi\right]\right. \\
& \left.-\sin \left[2 \pi\left(f_{S}+f_{R}\right) t+\varphi\right]\right\} .
\end{aligned}
$$

In most experimental situations, the signal is close in frequency to the reference: $f_{S}-f_{R} \ll f_{R}$ and $f_{S}+f_{R} \approx$ $2 f_{R}$. For the case $f_{S}=f_{R}$, we have $f_{S}-f_{R}=0$ and $f_{S}+f_{R}=2 f_{R}$, and the unfiltered $x$-channel and $y$-channel outputs contain a rapidly oscillating term superimposed on a time-independent one:

$V_{M X}=V_{I} v_{R X}=\frac{1}{2} g A_{S}\left\{\cos (\varphi)+\cos \left[2 \pi\left(2 f_{R}\right) t+\varphi\right]\right\}$,

$V_{M Y}=V_{I} v_{R Y}=\frac{1}{2} g A_{S}\left\{\sin (\varphi)-\sin \left[2 \pi\left(2 f_{R}\right) t+\varphi\right]\right\}$.

Finally, $V_{M X}$ and $V_{M Y}$ are each fed through a low-pass filter with a "time constant" $\tau=1 /\left(2 \pi f_{c}\right)$, where $f_{c}$ is the "cutoff" or "corner" frequency of the filter. As a rule of thumb, the filter preserves signals with frequency $f \ll f_{c}$, 
while attenuating signals with $f \gg f_{c}$. The resulting filtered outputs are defined as $V_{\text {Out } X}$ and $V_{\text {Out } Y}$ in the tutorial. In the idealized version of the case in which $f_{R}=f_{S}$, the time constant should be selected such that the low-pass filter attenuates the second-harmonic $\left(2 f_{R}\right)$ term from both $V_{M X}$ and $V_{M Y}$ resulting in a time-independent output signal. The relationship between $V_{\text {Out } X}$ and $V_{\text {OutY }}$ and the magnitude and phase of the input signal as initially defined is given by the following familiar trigonometric identities:

$$
\begin{aligned}
& V_{\text {Out } X}=\frac{1}{2} g A_{S} \cos \varphi, \\
& V_{\text {Out } Y}=\frac{1}{2} g A_{S} \sin \varphi .
\end{aligned}
$$

Or

$$
\begin{gathered}
A_{S}=(2 / g) \sqrt{V_{\text {Out } X}^{2}+V_{\text {Out } Y}^{2}}, \\
\varphi=\tan ^{-1}\left(V_{\text {Out } Y} / V_{\text {Out } X}\right) .
\end{gathered}
$$

As noted earlier, the most common use of a lock-in in laboratory research is to measure small signals that are synchronous with an external reference, often in the presence of large background signals or noise. For this class of measurements, the reference frequency is set equal to the signal frequency $\left(f_{R}=f_{S}\right)$. However, there are applications in which the two frequencies would not be the same; alternately, when changing a parameter that affects the signal, one is effectively modulating the signal in time. This type of "amplitude modulation" will produce sidebands around $f_{S}$ which must be passed with acceptably low attenuation, while making sure that the signal is not "flooded" with background noise. While the lock-in is most commonly used in the ideal case $\left(f_{R}=f_{S}\right.$ with the $2 f_{R}$ strongly attenuated) with no other superimposed signals, there are many instances in which unwanted signals can interfere with measurement. Power line noise (i.e., the 50 or $60 \mathrm{~Hz}$ frequency introduced by ac electrical power) can often superimpose large sinusoidal signals over the desired measurements. Additionally, there are a multitude of laboratory situations in which information can be gained from the amplitude and phase measured by the lock-in. For example, Cahill uses phase information from a lock-in to analyze time-domain thermoreflectance measurements of diffusion across interfaces and microfabricated structures [22]. A number of other examples can be found in Kremer and Schönhals' book, Broadband Dielectric Spectroscopy, which discusses how lock-ins can be used to measure the complex dielectric response of materials as a function of frequency [23]. We note that according to the faculty members who were interviewed during the development of the lock-in (discussed further in the Methodology section), what is particularly challenging for students is how to interpret the input and output for a particular measurement of the amplitude and phase of the signal. In particular, their graduate students in the lab know they have to measure the amplitude and/or phase of the signal using the lock-in in a particular context but they often have difficulty figuring out how to set the input parameters correctly or how to troubleshoot if the output looks anomalous. Thus, the goal of the tutorial is not to help students learn about why amplitude and/or phase of a signal may be useful, for example, for measuring complex dielectric response of a material as a function of frequency, but rather how to interpret the input and output signals in various situations. Thus, helping all students who use lockin amplifiers in any context to measure the amplitude and/ or phase of the signal to develop a functional understanding of the common uses (and misuses) of lock-in amplifiers represents the primary goal of the lock-in tutorial.

\section{METHODOLOGY}

Before the development of the tutorial and the associated pretest and post-test (pretest is administered before the tutorial and post-test after it), informal discussions with faculty members who conduct research in experimental condensed matter physics using the lock-in were carried out. These discussions provided information about the fact that even the graduate students, who have been using this device for some time, have many common difficulties in using this device effectively. Therefore, we decided to start the development of the tutorial by conducting audiorecorded interviews with several faculty members. We individually interviewed five physics professors at the University of Pittsburgh who conduct research in condensed matter physics and commonly work with graduate students who use lock-ins in their research. A typical interview time was 60-90 min, during which each professor was also asked to articulate what they expected their students to know about lock-ins, what was the goal of the professor's experimental research projects utilizing lockins, how lock-ins are useful in the broader framework of their research, and what were the common difficulties their students had with the device. Most commonly, student difficulties tended to arise from students lack of understanding of the fundamental operation of the lock-in and how the input signal was processed by the device to generate an output signal. This lack of understanding of the fundamental operation made it difficult if not impossible for students to troubleshoot common issues faced when making use of a lock-in. The faculty interviewed uniformly expressed an interest in their students having an increased understanding of why they are using the lock-in to take measurements as well as how this device functions to allow accurate amplitude measurements of a targeted frequency. Additionally, an interest in student ability to apply this understanding of the lock-in to troubleshoot 
problems was expressed and emphasized by anecdotal accounts of difficulties students working with these faculty members had encountered. Several faculty members also expressed interest in allowing students who are new to the lab setting an opportunity to develop a degree of comfort with the lock-in before their first interaction with one in a lab environment.

Throughout the course of these faculty interviews, five specific situations in which students had difficulties with the lock-in were mentioned frequently: (i) Students had little understanding of how the lock-in operated when the signal and reference frequencies are not equal. (ii) Faculty members were concerned that students did not understand that the phase difference between the signal and reference frequency could be examined by using a lock-in. (iii) Situations in which the amplitude of a low frequency measured could result in the $2 f_{R}$ signals passing into the output if the time constant is set too low. (iv) Faculty expressed that students should know how much time is required for the output signal to stabilize after a change to the input signal based on the time constant used. (v) Situations in which additional frequencies are present in the input signal and can disrupt the output signal if the time constant is set too low. In addition to the most common case discussed above and the general difficulties with lock-in that the faculty members mentioned, these five situations that are of great interest to them in the lab form the basis of the situations explicitly addressed in the tutorial. Further details of the initial difficulties students had with these cases that the faculty noted were important for students to have a clear understanding of and the effectiveness of the tutorial at addressing them will be discussed later in the results section.

Using the feedback from professors as a guide along with a cognitive task analysis of the underlying knowledge involved in the operation of a lock-in, we developed a preliminary tutorial along with a pretest and post-test. The tutorial first helps students understand the strengths and weaknesses of the lock-in with respect to other measurement devices that they may have encountered in the past, and then to provide them with a firm understanding of the components and principles that are used in this device to generate an accurate measurement of the amplitude. The tutorial strives to progressively expand students' knowledge of the fundamentals into intuitive understanding of what the lock-in's output informs about the input signal and vice versa. The pretest and posttest were developed to include problems which demonstrate an array of potential situations that may be encountered in a lab setting and probe students' knowledge of the fundamentals of the lockin. The different situations students learn using a guided inquiry approach in the tutorial and the associated pretest and posttest discussed in detail later were inspired by the insights obtained from faculty interviews about what their students used the lock-in for and what they struggled with.
The tutorial development was accompanied by the simultaneous development of a lock-in simulation. The simulation is an integral component of the tutorial. The simulation was integrated with the tutorial with the intent to give students an opportunity to experience the operation of the device from anywhere and develop a functional understanding about it using a proxy for the actual device.

At various stages of the development, we also interviewed graduate students who made use of the most up-todate version of the tutorial using a think-aloud protocol to better understand their difficulties and to fine-tune the tutorial as well as its associated pretest, post-test, and simulation. In these semistructured interviews, students were asked to talk aloud while they worked through the pretest, tutorial, and the posttest. The interviewer tried not to disturb students' thought processes while they answered the questions except to encourage them to keep talking if they became quiet for a long time. Later, the interviewer asked students for clarification of points they had not made clear earlier in order to understand their thought processes better. These interviews were semistructured in that some of these questions were planned out ahead of time while others were emergent queries based upon a particular student's responses to questions during an interview. A typical interview with a graduate student took between 2 and $3 \frac{1}{2}$ hours.

The tutorial (along with the pretest and post-test) was iteratively refined over 30 times, based upon feedback from graduate students and professors. After the tutorial and its supplementary material underwent this process of revision and fine-tuning, it was administered to 21 additional physics graduate students who had not been involved in the development phase of the tutorial. These students ranged in experience from those who had been introduced to the basics of the lock-in in their research group but had not made use of one for their own research to those with extensive experience with the lock-in and either concurrently used a lock-in for their research or had made extensive use of one in the past.

After students completed the post-test they were asked if they thought that the tutorial had improved their understanding of the lock-in as well as whether they would recommend it to another student who either currently worked with or was about to begin working with lockins. All students interviewed noted that they felt that their understanding of the device had been greatly improved and that they would recommend the tutorial to students in the future. Additionally, several of the students interviewed explicitly requested copies of the tutorial for their own use and/or for the use of others that they worked with. The faculty members with whom the tutorials were iterated were generally very enthusiastic about the final version of the tutorial. They felt that the tutorial would be very helpful for students using this device in their lab. One faculty member even expressed interest in the potential to use the 
tutorial in his advanced undergraduate lab due to the ground-up approach taken in the tutorial, starting with the fundamentals and gradually helping students develop a solid understanding so that they can even tackle advanced situations and learn to troubleshoot in various situations.

As noted, to evaluate the effectiveness of the tutorial a pretest and post-test were developed. A generalized grading rubric (summary in Table I) was developed which is capable of grading questions with all of the possible outcomes that could result when solving each of the problems on the pretest and post-test involving the operation of the lock-in. To this end, three researchers jointly deliberated a series of six classes of rubrics that could be used in the scoring of all problems present in all versions of the pretest and post-test. The final version of the rubric resulted in better than $90 \%$ interrater reliability between two researchers for the scoring of performance on the pretest and post-test questions.

The tutorial uses a guided approach to learning to help students develop a solid understanding of lock-in and the ability to apply this understanding to the many varied situations that can arise when using this device. Appropriate feedback and scaffolding support is provided as needed. In order to help students develop a good grasp of the functioning of the device, the tutorial integrates the conceptual and mathematical aspects of the lock-in. Students first learn to make sense of simple situations, but as the tutorial progresses, they steadily learn to analyze more complex situations which were inspired by discussions with the faculty members. Learning to analyze these diverse situations can help students develop a nuanced understanding of the device and be able to troubleshoot as needed. Several examples in the tutorial explicitly ask students to synthesize their learning from two or more prior situations to come up with a prediction for the next case. The scaffolding support and feedback provided in the tutorial is such that all students, regardless of their prior experience with lock-in, can benefit from the tutorial. Below, we discuss the common difficulties found via research before discussing the structure of the researchbased tutorial and results from the pretest and post-test administered before and after students worked on the tutorial, respectively.

\section{STUDENT DIFFICULTIES}

In addition to the faculty members' feedback, throughout student interviews, many common difficulties that graduate students have in using the lock-in were identified when they were asked to make predictions about either the output signal or input signal of the lock-in in diverse situations. We observed that, before working on the tutorial, most of the interviewed graduate students had an incomplete understanding of the intricacies of the lock-in's operation and many of the interviewed students, even those with extensive experience using the lock-in to make measurements in a lab setting, were only capable of predicting the input or output for the simplest of cases. The difficulties found in our investigation have roots in a lack of coherent understanding of the fundamentals of a lock-in. For example, students often had a fuzzy understanding of what the mixers in a lock-in do. Even in the cases that are most typically encountered in the lab setting, interviewed students often demonstrated only a superficial understanding of the lockin. The range and prevalence of these difficulties during the interviews demonstrates that students are often using the lock-in as a black box to make measurements at a targeted frequency without understanding the intricacies of its operation. They are unlikely to troubleshoot difficulties as many of the interviewed faculty members had already pointed out during their interviews. The following are difficulties commonly observed among interviewed students before exposure to the tutorial or in the early portions of the tutorial as these difficulties were encountered for the first time.

\section{A. Difficulty in calculating and using the cutoff frequency}

Interviews suggest that one major aspect of the lock-in that is often overlooked by graduate students is the effect of the low-pass filter on the output signal. For example, interviewed students had great difficulty with the fact that the frequencies that will make it into the output signal can be estimated by making use of the time constant, $\tau$. Many students ignored the time constant throughout the pretest with some commenting that they didn't know how to account for the time constant. For example, one student stated during a think-aloud interview while working through the tutorial that "The most helpful part [of the tutorial] is [when] it explains the [low-pass] filter. That is a part I seldom use in the lock-in." The student went on to explain that he didn't understand how to set the time constant and had little understanding of its purpose in the lock-in despite having worked with the device for roughly a month for authentic research. This confusion regarding the effect of the time constant results in students being unable to properly predict what frequencies will make it through the low-pass filter with little attenuation and thus the frequencies that will appear in the output signal. This difficulty is not directly measured by any of the pretest and post-test problems but thorough understanding of this concept is required to solve all pretest and post-test problems.

\section{B. Difficulty with the case in which $f_{S}=f_{R}$ and the $2 f_{R}$ signal is strongly attenuated}

Since the lock-in amplifier is most commonly used to measure the amplitude of a targeted frequency present in an input signal, the case in which the signal and reference frequency are equal and the time constant is high enough to attenuate the $2 f_{R}$ signal is the most familiar to most of 
TABLE I. Summary of the grading rubrics used for the pretest and post-test questions discussed. Rubrics 1 through 3 are used to score problems in which the students are asked to sketch the output signal for a given set of input parameters (output problems) and rubrics 4 through 6 are used to score problems in which the students are asked to provide the input parameters for a given output (input problems). Case 1 problems are scored by rubric 1 for output problems and rubric 4 for input problems. Case 2 problems are scored by rubric 2 for output problems and rubric 4 for input problems. Case 3 problems are scored by rubric 1 for output problems and rubric 5 for input problems. Case 4 problems are scored by rubric 3 for output problems and rubric 4 for input problems. Case 5 problems are scored by either rubric 1 or rubric 3 for output problems, depending on the time constant and amplitude modulation frequency given in the problem. There are no case 5 problems in which output is provided and students are asked for input parameters. Case 6 problems are scored by rubric 3 for output problems and rubric 6 for input problems.

Rubric 1: Correct answer should have no frequency and nonzero dc offset present in the output signal.

\begin{tabular}{|c|c|c|c|c|}
\hline \multirow{4}{*}{$\begin{array}{l}\text { Nonzero frequency } \\
\text { present? }\end{array}$} & Yes & 0 points & & \\
\hline & \multirow[t]{3}{*}{ No } & \multirow{3}{*}{$\begin{array}{l}\text { Magnitudes of } \\
\text { dc components } \\
(x, y) \text { are? }\end{array}$} & Correct & 10 points \\
\hline & & & Incorrect but nonzero & 5 points \\
\hline & & & Zero & 0 points \\
\hline
\end{tabular}

Rubric 2: Correct answer should have a nonzero frequency and zero dc offset present in the output signal.

\begin{tabular}{|c|c|c|c|c|c|}
\hline \multirow[t]{2}{*}{$\begin{array}{l}\text { Nonzero frequency } \\
\text { present? }\end{array}$} & \multirow[t]{2}{*}{ Yes } & \multirow{2}{*}{$\begin{array}{l}\text { Magnitudes of both } \\
\text { dc components } \\
(x, y) \text { are zero? }\end{array}$} & Yes & 5 points & $\begin{array}{l}+2.5 \text { points for } \\
\text { correct frequency }\end{array}$ \\
\hline & & & No & 2.5 points & $\begin{array}{l}+2.5 \text { points for } \\
\text { correct amplitude }\end{array}$ \\
\hline & No & 0 points & & & \\
\hline \multicolumn{6}{|c|}{ Rubric 3: Correct answer should have a nonzero frequency and nonzero dc offset present in the output signal. } \\
\hline \multirow[t]{4}{*}{$\begin{array}{l}\text { Nonzero frequency } \\
\text { present? }\end{array}$} & \multirow[t]{2}{*}{ Yes } & \multirow{2}{*}{$\begin{array}{l}\text { Magnitudes of both } \\
\text { dc components } \\
(x, y) \text { are correct? }\end{array}$} & Yes & 5 points & $\begin{array}{l}+2.5 \text { points for } \\
\text { correct frequency }\end{array}$ \\
\hline & & & No & 2.5 points & $\begin{array}{l}+2.5 \text { points for } \\
\text { correct amplitude }\end{array}$ \\
\hline & \multirow[t]{2}{*}{ No } & \multirow{2}{*}{$\begin{array}{l}\text { Magnitudes of both } \\
\text { dc components } \\
(x, y) \text { are correct? }\end{array}$} & Yes & 2.5 points & \\
\hline & & & No & 0 points & \\
\hline
\end{tabular}

Rubric 4: Correct answer should have one frequency in the input signal.

\begin{tabular}{|c|c|c|c|c|c|}
\hline \multirow[t]{4}{*}{ Frequency correct? } & \multirow[t]{2}{*}{ Yes } & \multirow[t]{2}{*}{ Amplitude correct? } & Yes & 10 points & \multirow{4}{*}{$\begin{array}{l}\text { Divide score by } 2 \text { if a second } \\
\text { input signal is given that } \\
\text { - would result in a different } \\
\text { output signal. }\end{array}$} \\
\hline & & & No & 5 points & \\
\hline & \multirow[t]{2}{*}{ No } & \multirow[t]{2}{*}{ Amplitude correct? } & Yes & 5 points & \\
\hline & & & No & 0 points & \\
\hline
\end{tabular}

Rubric 5: Correct answer should have one frequency in the input signal with a phase angle.

\begin{tabular}{llllll}
\hline Frequency correct? & Yes & Amplitude correct? & Yes & 5 points & +5 points for correct phase \\
& & & No & 2.5 points & $\begin{array}{l}\text { angle Divide score by } 2 \text { if a } \\
\text { second input signal }\end{array}$ \\
\cline { 2 - 5 } & No & Amplitude correct? & Yes & 2.5 points & $\begin{array}{l}\text { is given that would result } \\
\text { in a different } \\
\text { output signal. }\end{array}$ \\
\cline { 3 - 5 } & & No & 0 points & & \\
\hline
\end{tabular}

Rubric 6: Correct answer should have two frequencies in the input signal.

\begin{tabular}{|c|c|c|c|c|c|}
\hline \multirow[t]{4}{*}{ Frequency correct? } & \multirow[t]{2}{*}{ Yes } & \multirow[t]{2}{*}{ Amplitude correct? } & Yes & 5 points & \multirow{4}{*}{$\begin{array}{l}\text { Use the rubric for each } \\
\text { of the two frequencies that } \\
\text { should appear in the } \\
\text { input signal } \\
\text { and sum the points. }\end{array}$} \\
\hline & & & No & 2.5 points & \\
\hline & \multirow[t]{2}{*}{ No } & \multirow[t]{2}{*}{ Amplitude correct? } & Yes & 2.5 points & \\
\hline & & & No & 0 points & \\
\hline
\end{tabular}


those who have used the lock-in in a lab setting. Despite this being the most common case, some students still had difficulty with predicting the output signal from a lock-in under these conditions. These students most commonly incorrectly thought that the lock-in's output should have a frequency equal to that of the signal frequency. They thought that the lock-in is providing an output similar to that which would be shown by an oscilloscope measuring the input signal. This difficulty demonstrates a misunderstanding of the operation of the lock-in for these conditions. Since the purpose of the lock-in is to measure the amplitude of the input signal at the targeted frequency (determined by the reference frequency) by multiplying the input signal to a dc output, a frequency cannot be present in the output when $f_{S}=f_{R}$ and the $2 f_{R}$ signal is strongly attenuated. This difficulty is assessed by questions from case 1 in the pretest and post-test discussed below.

\section{Difficulty with the case in which $f_{S} \neq f_{R}$, the $f_{R}-f_{S}$ signal is not strongly attenuated and the $f_{R}+f_{S}$ signal is strongly attenuated}

This difficulty with the operation of the lock-in amplifier affects the student's ability to make predictions regarding other lock-in setups. In the case in which $f_{S} \neq f_{R}$ students demonstrated several difficulties rooted in a lack of conceptual understanding of the operation of the lock-in. One common difficulty among students when confronted with this situation involved students stating that the lock-in would not show an output from any frequency in the input signal that does not equal the reference frequency. These students commonly determined that both the $x$ and $y$ output of the lock-in would be zero. It is worth noting that this result was often due to students commonly disregarding the time constant provided in the question statement. For example, one student demonstrated this difficulty when he said "So there's a frequency difference between them (the reference and signal frequency)... So I expect zero for this [output] although the frequencies are close." It appears that the student is considering the effect of the reference and signal frequency being close to one another. Despite this consideration, the student selected zero as the output for both channels. Additionally, this student went on to say "...the entire point of the lock-in is that it should pick out only the frequency... only the component of the same frequency as the reference" a few minutes later in the interview, demonstrating that he also thought that the lockin is only capable of measuring input signals with a frequency equal to the reference frequency.

Another common difficulty among students is that many thought that the lock-in will yield a dc output even under the condition $f_{S} \neq f_{R}$. These students came up with an output that would have been correct if the signal and reference frequency had been equal to each other rather than an output with a frequency of $\left|f_{S}-f_{R}\right|$ present in it with effectively no attenuation from the low-pass filter.
This difficulty is partly due to their lab experience being primarily focused on the most common case. Since some students only make use of the lock-in as a measurement device under the condition that $f_{S}=f_{R}$ they have difficulty with any situation that causes the lock-in's output to be anything but a dc signal. Thus, many of these students thought that the output signals from a lock-in must always be a dc signal. Interviews suggest that this type of incorrect rote application is often due to the fact that students are using the lock-in as a black box rather than understanding the operation of this device before making use of it. This type of difficulty can prevent students from correctly troubleshooting if they encounter unexpected output in their lab experiments. This difficulty is related to faculty concern 1 and is measured by questions from case 2 in the pretest and post-test discussed below.

\section{Difficulty with the case in which $f_{S}$ and $f_{R}$ are out of phase $(\varphi \neq 0)$}

The most common difficulty that students had with the nonzero phase involves cases in which $f_{S} \neq f_{R}$, the $f_{R}-f_{S}$ signal is not strongly attenuated, and the $f_{R}+f_{S}$ signal is strongly attenuated. Students would commonly combine factors of the correct solution to this case with factors that are used to solve the case where $f_{S}=f_{R}$ and $\varphi \neq 0$. Most commonly, this resulted in a time varying output with different amplitudes for the $x$-channel and $y$ channel outputs. Students would often determine the amplitude of the $x$ channel to be $A_{S} \cos (\varphi)$ and the amplitude of the $y$ channel to be $A_{S} \sin (\varphi)$, rather than $\varphi$ only determining the starting point of the $x$-channel and $y$-channel outputs. Additionally, students showed weaker performance on problems in which $f_{S}=f_{R}$ and $\varphi \neq 0$. This difficulty is related to faculty concern 2 and is measured by questions from case 3 in the pretest and post-test discussed below.

\section{E. Difficulty with the case in which $f_{S}=f_{R}$ and the $2 f_{R}$ signal is unattenuated}

Another situation that caused a considerable amount of difficulty among students is when $f_{S}=f_{R}$ and the $2 f_{R}$ signal appears in the output. Most commonly, students answering this question provide a dc output without a $2 f_{R}$ signal. The difficulty in answering this type of question stems from two sources that have already been mentioned. First is a student making use of the lock-in as a black box for the most common case, in which they observe a dc output if they chose a sufficiently high time constant when using the device. Additionally, the students' lack of understanding of the time constant and how it affects the attenuation of signals in the output signal often leads them into thinking that the low-pass filter will remove all frequencies in the output signal.

In addition to these two common difficulties, we find that many students had an even more fundamental issue with the 
operation of the lock-in that led to difficulty with this case. In particular, many students lacked a coherent understanding of the internal operation of the lock-in and how the multiplication of the input signal and reference signal results in a sum and difference frequency in the output signal. Most of the students interviewed showed an understanding of the difference frequency which resulted in a dc output in this case but failed to even mention the existence of the sum frequency which would result in the $2 f_{R}$ signal in this case. Additionally, none of the students interviewed made use of the relevant equations for the multiplication of the input and reference signals in the mixer to gain insight into what output they should expect when the questions were posed in the pretest. This difficulty is related to faculty concern 3 and is measured by questions from case 4 in the pretest and post-test discussed below.

\section{F. Difficulty with amplitude modulation of the input signal}

Another case in which students had difficulty during the think-aloud interview is the introduction of an amplitude modulation to the primary input frequency. The most common difficulty observed during interviews is that students often thought that the amplitude modulation would affect both the $x$-channel and $y$-channel output signal even when the amplitude only affects the output in one of the two channels (such as when the phase of the input signal $\varphi$ is either $0^{\circ}$ or $90^{\circ}$ ). Interviews suggest that this difficulty is due to a combination of a lack of experience with amplitude modulation paired with the students' experience with other cases that introduce frequencies into the output signal. For all other cases that result in frequencies in the output signal of the lock-in, the frequency appears in both channels of the output with equal amplitude. This prior experience colors the students expectations about the possible output signal of the lock-in. Many students also had difficulty determining how the low-pass filter affects the amplitude modulation. Despite amplitude modulation generating a frequency that is passed through the low-pass filter in the same manner as any other frequency, some students either thought that amplitude modulation should not be affected by the low-pass filter or completely ignored consideration of the effects of the low-pass filter on this signal. This difficulty is related to faculty concern 4 and is measured by questions from case 5 in the pretest and post-test discussed below.

\section{G. Difficulty with multiple frequencies present in the input signal}

One final aspect of the lock-in that practically all interviewed students had difficulty with was the case in which multiple frequencies are present in the input signal. Interviews suggest that many students had not considered such a possibility in the past. Although the difficulty with this case was common to all students interviewed, their reactions to this problem during interviews were often varied with only one common type of student response. Most commonly, students ignored one of the two frequencies and continued to treat the other frequency as though it was the only one in the input signal. One student demonstrated this confusion with the statement "So, since I don't really know how to incorporate the changing (indicates the additional signals section) into it, I'm just going to stick with the amplitude and phase (indicates the primary input signal section)." Thus, when this student faced a situation that he did not have experience with, he opted to ignore the source of confusion rather than find a way to incorporate it into the output signal. This tendency can have a detrimental effect on student ability to troubleshoot as was already pointed out by the interviewed faculty members in actual situations in research labs. This difficulty is related to faculty concern 5 and is measured by questions from case 6 in the pretest and post-test discussed below.

The last five of these seven student difficulties are directly aligned with the five situations discussed in faculty interviews in which students have difficulty. Of the remaining two (student difficulties 1 and 2), the first is an underlying issue that may be partially responsible for a number of the other difficulties observed, and the second is likely not noticed as commonly by faculty as most of the students in their labs had become relatively adept at using the lock-in as a black box. Student difficulty 2 only became apparent when prompting students to sketch lock-in outputs for this most commonly encountered case, which is a particularly uncommon practice in the laboratory setting. From this mapping, it appears that faculty had a reasonable understanding of student difficulties as they are manifested under relatively commonly encountered laboratory conditions but had several gaps associated with the underlying causes and issues associated with student understanding and cases where students had learned enough to appear knowledgeable despite their lack of understanding.

\section{THE TUTORIAL STRUCTURE}

The most up-to-date version of the tutorial, simulation, and the associated pretest and post-test are available for download on ComPADRE [24]. As noted earlier, students first take a pretest before working on the tutorial. The pretest takes the form of a short quiz comprised of questions related to the function of a lock-in that the faculty members using the lock-in in their lab were interest in. These questions are posed making use of the interface of the simulation which was developed alongside the tutorial. For each of the questions, the student is provided with a screenshot of the simulation's interface with either the output erased or certain components of the input signal removed and the student is asked to provide the missing information (as seen in the pretest and post-test [24]). These questions, while superficially similar in structure, widely vary in difficulty depending upon which information is 
available and how the values provided interrelate (analogous to the varying difficulty in Sudoku puzzles depending upon initially provided values). Many of these problems engage students at the synthesis level of Bloom's taxonomy. These problems are divided amongst six cases (which will be described in detail later) with each of these six cases directly assessing whether students have difficulties 2-7 described earlier. Student difficulty 1 is also assessed via each of these problems when determining which frequencies will pass into the output signal unattenuated. Students are provided with a sheet that contains supplementary information to aid them as they work through this pretest. This supplementary information was developed to ensure that student performance on the pretest was not the result of confusion about the form in which the questions were asked or a lack of understanding of some of the terms and information used in these pretest questions.

After the students complete this pretest, they work through the tutorial. The tutorial begins by guiding students to perform a brief comparative analysis of several other measurement devices (the voltmeter, oscilloscope, and spectrum analyzer) in addition to the lock-in followed by an in-depth examination of the dual-channel lock-in which begins with a short narrated video followed by a detailed explanation of a diagram of the dual-channel lock-in. The student is then guided to find the mathematical expressions for $V_{M X}$ and $V_{M Y}$ both in the general case (when $f_{S} \neq f_{R}$ ) and in the most typical case (when $f_{S}=f_{R}$ ). Integration of conceptual and quantitative aspects of lock-in is central to obtaining a functional understanding of the device. Particular attention is paid to having students focus on which frequencies will be in the unfiltered output signals for each of these two cases to ensure that students internalize both the sum frequency $\left(f_{S}+f_{R}\right)$ and difference frequency $\left(\left|f_{S}-f_{R}\right|\right)$. This is followed by having students learn the workings of the low-pass filter. The gradual explanation of the low-pass filter begins with a diagram of the amplitude loss with respect to the frequency for the low-pass filter for 6,12 , and $24 \mathrm{~dB} /$ Oct roll-off. While learning about these, the students are asked to note two rules of thumb for the $12 \mathrm{~dB} /$ Oct roll-off, which is used throughout the remainder of the tutorial. For all frequencies $f \leq 0.1 \times \tau^{-1}$ the students are asked to assume that there is practically no attenuation of the signal as it passes through the filter, while for all frequencies $f \geq 10 \times \tau^{-1}$ the amplitude is strongly attenuated. This treatment of the low-pass filter is performed in a very deliberate and methodical manner because student understanding of the cutoff frequency was found to be particularly deficient as discussed under student difficulty 1.

After learning about the basic treatment of the low-pass filter, students move on to make use of the simulation to solidify concepts. The simulation allows the students to manipulate all of the settings commonly found on a lock-in as well as modifying the characteristics of a simulated input signal. The simulation component of the tutorial begins by familiarizing students with the interface used by the simulation in a similar manner to the pretest supplement. Additionally, students are led through an example of how to predict the output signal of the lock-in by first applying the equations derived earlier in the mathematical treatment of the mixer and then applying the rules of thumb regarding the low-pass filter to provide an additional point of practice for student difficulty 1 . The students then work through a series of problems designed to be used with the simulation similar to the one in Fig. 1 (but in different contexts). For each problem, the student is asked to predict the output signal, sketch their predictions, and compare their sketch to the simulation output. After each prediction and simulation

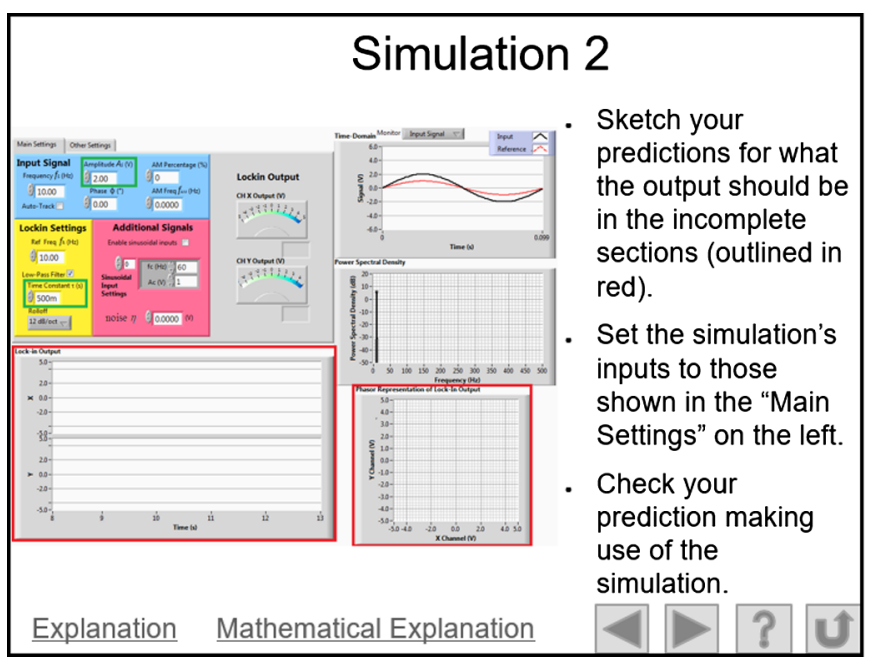

Simulation 2 Purpose This simulation demonstrates a very similar
case to that shown in simulation 1 . This
simulation serves to demonstrate one of the
most common uses of the lock-in amplifier.
This is to measure the amplitude of a specific
frequency in the input signal by setting $f_{S}=f_{R}$.

FIG. 1. Example of a typical simulation question (left) and the associated "purpose" slide (right). For the simulation question, red bounding boxes indicate information that the student is expected to sketch or provide, consistent with the lock-in settings and/or output and green bounding boxes highlight settings that were changed from a prior simulation question. 


\section{Explanation}

Since $f_{S}=f_{R}$, the multiplication of the input signal and reference signal yields both a constant and a $20 \mathrm{~Hz}$ component to the mixer output signal to be fed to the low-pass filter. Since $\tau=0.5 \mathrm{sec}$, the cutoff frequency is $\frac{1}{\pi} \mathrm{Hz}$ and the $20 \mathrm{~Hz}$ signal is almost completely attenuated after passing through the low-pass filter. This yields the constant output signal of amplitude $2 \mathrm{~V}$ that you observe in the simulation.

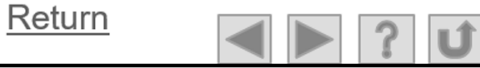

\section{Mathematical Explanation}

$$
\begin{gathered}
V_{M X}=2 V[1+\cos (2 \pi(20 \mathrm{~Hz}) t)] \\
V_{M Y}=-2 V[\sin (2 \pi(20 \mathrm{~Hz}) t)]
\end{gathered}
$$

unattenuated frequency $<0.1 \times(0.5 s)^{-1}=0.2 \mathrm{~Hz}$ attenuated frequency $>10 \times(0.5 s)^{-1}=20 \mathrm{~Hz}$

$$
\begin{gathered}
V_{\text {OutX }}=2 \mathrm{~V} \\
V_{\text {outY }}=0
\end{gathered}
$$

\section{$\underline{\text { Return }}$}

FIG. 2. Example of the explanations provided to students for each simulation (simulation 2 in this case). The "explanation" (left) features a conceptual description of the problem's solution while the "mathematical explanation" (right) features the mathematics required to solve the problem.

to scaffold student learning both a mathematical and a more conceptual intuitive explanation of the output signal based on the input parameters are provided as shown in Fig. 2, in case the students cannot reconcile their predicted output with the output shown on the screen in the simulation. In Fig. 2, the conceptual explanation describes the reasons for the output obtained without explicitly working out the mathematics associated with the mixer or the low-pass filter rules of thumb. The mathematical explanation, on the other hand, provides the mathematics used to determine the pre low-pass filter signal, the low-pass filter rules of thumb, and finally the filtered output signal. Throughout these simulation problems, students are led through a series of problems that address all of the student difficulties outlined above. While difficulties 2-7 are each explicitly addressed by one or two simulation problems that directly confront the underlying difficulty, difficulty 1 is addressed in all simulation problems via the act of determining which frequencies will be attenuated and which will not for each problem.

Following these simulation questions, the student works on a second set of questions that ask them to identify characteristics of either the input signal or the lock-in's settings by examining the provided output signal as shown in Fig. 3. The student is either asked to fill in the blank components of the input signal or asked to answer a multiple choice question regarding one of these parameters. Throughout these questions, which build on each other, several hints in the form of guiding statements or questions are provided to scaffold student learning (for each of these
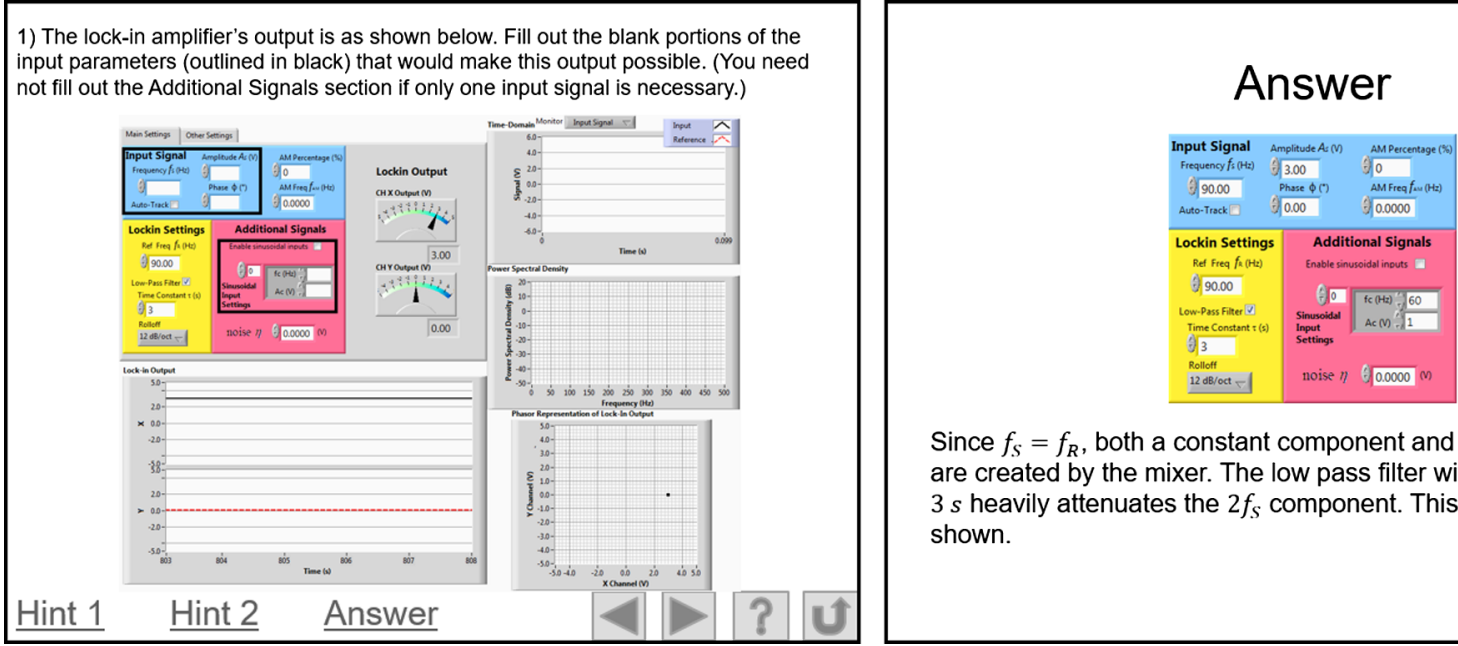

Since $f_{S}=f_{R}$, both a constant component and a $2 f_{S}$ component are created by the mixer. The low pass filter with a time constant of $3 s$ heavily attenuates the $2 f_{s}$ component. This yields the output shown.

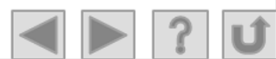

FIG. 3. An example of one of the tutorial questions which asks students to predict the input signal characteristics (left) and the answer to that question (right). 


\section{Hint 1}

The following discussion takes place between two students attempting to work out this problem.

Student 1: The only frequency in the input signal that can create a $\mathrm{DC}$ component in the output is one that equals the reference frequency. If this condition isn't met, the output shown cannot occur.

Student 2: I don't agree. The signal could have a frequency close enough to the reference frequency such that it is not filtered out of the output. This case could also yield a DC output like that shown here.

With which student do you agree?

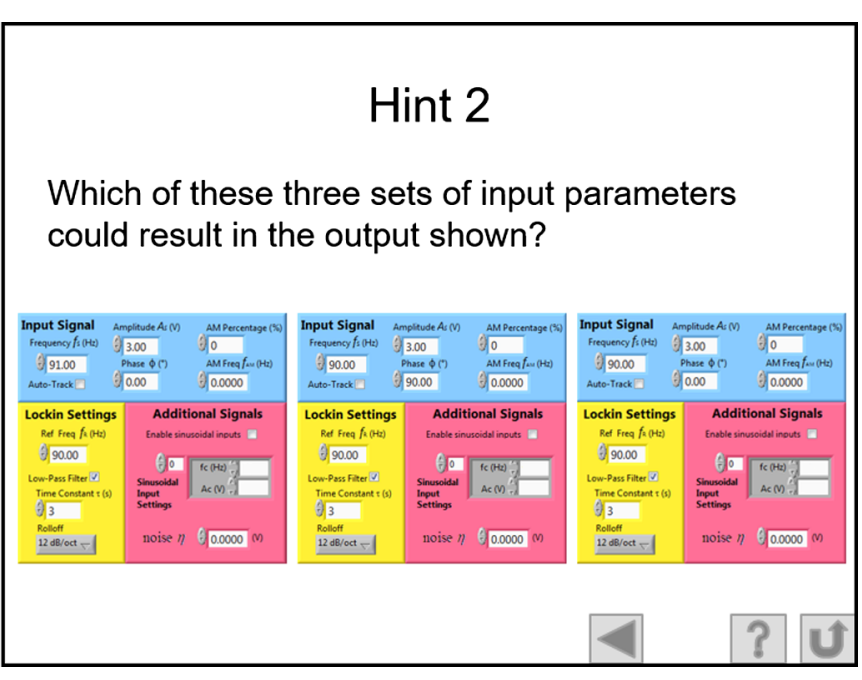

FIG. 4. Examples of two types of hint commonly provided to scaffold student learning.

questions as in Fig. 4). One type of hint commonly provided to the student as a first hint takes the form of a hypothetical discussion between two students that are attempting to solve that problem. This hint provides two viewpoints on that problem, one of which is correct and one of which is incorrect, for the student to consider. A more heavily scaffolded second hint commonly takes the form of providing three possible answers with minor differences for the student to consider. This allows the student to consider how each variation in the input signal results in a different output signal. Using a more lightly scaffolded hint first, followed by a more heavily scaffolded hint, allows the student to develop self-reliance as they become less dependent upon the hints (especially the heavily scaffolded hint) as they work through the tutorial, enabling the student's reliance on the scaffolding to fade [25]. After the students have answered each question to the best of their ability, the correct answer is provided along with an explanation for why that answer must be correct (see Fig. 3). The guiding problems that build on each other (and become more complex later) allow the student an opportunity to predict the outcome of the experiment in a given situation and determine input parameters and lock-in settings based on the output signal or output parameters for input settings. Similar to the simulation problems, each of these problems directly address one of the student difficulties (student difficulties 2-7) and provide an opportunity to solidify their understanding of the time constant and how it relates to the frequencies found in the output signal.

The post-test is structured in a similar manner to the pretest, consisting of a short quiz comprised of questions that demonstrate the same cases as in the pretest. Students are again allowed to have access to the same supplementary material for the post-test as in the pretest. To ensure that any change in score between the pretest and post-test was solely the result of the tutorial and not a difference in the specific problems used in each quiz, the questions used on the pretest and post-test are switched for roughly half of the students tested.

A more detailed description of the elements that make up this tutorial can be found in the prior publication which focuses more on the structure of the tutorial [21].

\section{RESULTS FROM THE PRETEST AND POST-TEST}

To evaluate the effectiveness of the tutorial at addressing these difficulties, a pretest and post-test were given to all students who worked on the tutorial. The difficulties discussed in the previous section were determined by examining student performance on the pretest as well as the tutorial and asking them for clarification of the points they had not made clear by themselves during the individual interviews (in which they first worked on the pretest, then they worked on the tutorial and then took the post-test).

In order to examine student understanding, six potential configurations of lock-in settings were included in the pretest and post-test questions. In the following section, each of these cases will be discussed in detail along with an examination of the tutorial's effectiveness at addressing student difficulties related to each case.

As noted earlier, three researchers jointly deliberated a series of six classes of rubrics that could be used in the scoring of all problems present in all versions of the pretest and post-test. Three rubrics were required to cover all possible output signals present in the pretest and post-test that could be achieved with either zero or nonzero amplitude dc components and zero or nonzero amplitude time varying components, and three were required for possible input signals with either one frequency, one frequency with a nonzero phase angle, or two frequencies present in the input signal. A summary of the rubrics that covers all cases can be found in Table I. Also, during the 
TABLE II. Summary of the average score, standard deviation and number of instances of each of these cases present in both the pretest and post-test.

\begin{tabular}{lccccrr}
\hline \hline & $\begin{array}{c}\text { Average pretest } \\
\text { score }\end{array}$ & $\begin{array}{c}\text { Pretest standard } \\
\text { deviation }\end{array}$ & $\begin{array}{c}\text { Number of } \\
\text { instances } \\
\text { in pretest }\end{array}$ & $\begin{array}{c}\text { Average post-test } \\
\text { score }\end{array}$ & $\begin{array}{c}\text { Post-test standard } \\
\text { deviation }\end{array}$ & $\begin{array}{c}\text { Number of } \\
\text { instances } \\
\text { in post-test }\end{array}$ \\
$p$ value \\
\hline Case 1 & $76.4 \%$ & $40.6 \%$ & 34 & $100 \%$ & $0.0 \%$ & 33 \\
Case 2 & $22.0 \%$ & $34.7 \%$ & 33 & $90.9 \%$ & $21.2 \%$ & 0.002 \\
Case 3 & $55.9 \%$ & $41.3 \%$ & 36 & $89.2 \%$ & $24.7 \%$ & 33 \\
Case 4 & $33.0 \%$ & $32.3 \%$ & 22 & $68.2 \%$ & $30.3 \%$ & $<0.001$ \\
Case 5 & $26.2 \%$ & $32.3 \%$ & 21 & $83.0 \%$ & $34.9 \%$ & 22 \\
Case 6 & $25.0 \%$ & $38.4 \%$ & 33 & $80.3 \%$ & $33.6 \%$ & $<0.001$ \\
\hline \hline
\end{tabular}

development and evaluation of the tutorial, a few additional pretest and post-test questions were included in the later version. This accounts for the varying number of instances of a particular case present in the pretest and post-test shown in Table II. Below, we describe each case.

\section{A. (Case 1) $f_{R}=f_{S}$ with the $2 f_{R}$ signal strongly attenuated and $\varphi=0$}

This case is the most commonly encountered lab situation involving the lock-in in which students are attempting to measure the amplitude of the frequency $f_{S}$ in the input signal and have correctly set the reference frequency equal to $f_{S}$ and the time constant high enough to filter out the $2 f_{R}$ signal. Despite this case being one that should be typical to students who commonly make use of this device, some students had difficulty with these problems. Examining Table II, we can see that the average score across all pretest problems that demonstrate this case is roughly $75 \%$. This not-perfect performance results from some students having only limited experience with the lock-in as well as some experienced students who thought that the lock-in would provide an output with frequency $f_{R}$ in this case as already discussed earlier. While these pretest results are not particularly disheartening, as shown in Table II, the tutorial does show a marked improvement in student performance with every student that made use of the tutorial answering every question on the posttest that demonstrated this case perfectly $(100 \%)$. This demonstrates the effectiveness of this tutorial at ensuring that all students understand how the lock-in operates in this simple case.

\section{B. (Case 2) $f_{R} \neq f_{S}$, the $f_{R}-f_{S}$ signal is not strongly attenuated and the $f_{R}+f_{S}$ signal is strongly attenuated}

This case is less commonly encountered by students in the lab setting. This situation could arise if the student meant to measure the amplitude of frequency $f_{S}$ in the input signal by setting $f_{R}=f_{S}$ and incorrectly sets $f_{R}$ to a value near $f_{S}$. This situation also serves to illustrate the importance of integrating conceptual understanding with mathematical preliminaries that are central to the lock-in's operation. By correctly answering questions that demonstrate this case, students have to apply both the correct general equations for the unfiltered output signal as well as the effect of the lowpass filter for the time constant and roll-off designated for the problem. Examining Table II, we can see a distinct lack of student understanding on the pretest with the average student score being only slightly over $20 \%$ across all pretest problems that demonstrated this case. This lack of understanding is based partly in the interviewed students only rarely encountering this case. Throughout the pretest, most students were only able to correctly answer problems that were similar to the most common case. Student responses in interviews illustrate that students use the lock-in as a black box rather than by identifying the output signal based on a deep understanding of the internal operation of the lock-in. After working through the tutorial, student performance improved to an average of roughly $90 \%$. This improvement was primarily the result of improved student understanding of the mixer as well as the use of the rules of thumb to deal with the low-pass filter.

\section{C. (Case 3) $f_{R}=f_{S}$ with the $2 f_{R}$ signal strongly attenuated and $\varphi \neq 0$}

This case is very similar to case 1 (the most commonly faced lab situation) with the only difference being that the phase angle is no longer set to zero. This case could commonly be faced by students in the lab setting when the frequency of interest, $f_{S}$, is out of phase with the reference frequency. This case also served to illustrate the importance of having both an $x$-channel and $y$-channel output, which is an issue that was confusing to several students during interviews before they worked on the tutorial. This case shows that the amplitude of the frequency, $f_{S}$, cannot, in general, be measured completely in either the $x$ channel or $y$ channel without the reference frequency being in phase with $f_{S}$. Students showed decreased performance on these problems when compared to case 1 scoring slightly over $55 \%$ on average on the pretest. This is partly the result of students having somewhat less experience with this case than with the case in which $f_{S}$ is in phase with $f_{R}$, due to students commonly having control over the phase of the reference frequency in most lab experiments involving the 
lock-in. This average was improved to nearly $90 \%$ after working on the tutorial.

\section{D. (Case 4) $f_{R}=f_{S}$ with the $2 f_{R}$ signal not strongly attenuated and $\varphi=0$}

This case is primarily experienced by students when they are measuring a frequency $f_{S}$, which is very small and the time constant is incorrectly set resulting in the $2 f_{R}$ signal being passed by the low-pass filter. This case is used to demonstrate the importance of correctly accounting for the effect of the low-pass filter and selecting a time constant that will properly limit the frequencies that can pass. Students had little experience with this case before the tutorial leading to a very low average pretest score of under $35.0 \%$. The actual student understanding of the $2 f_{R}$ signal passing through the filter is even lower than the average pretest score, which suggests with most of the credit earned on problems that demonstrate this case come from correctly determining the dc offset of the output in accordance with rubric 3 in Table I. The tutorial was able to improve student understanding such that the average post-test score was roughly $70 \%$. The relatively lower improvement demonstrated in this case when compared to the others is partly the result of the fact that in all other cases the larger of the two frequencies generated by the multiplier is strongly attenuated while passing through the low-pass filter. This leads to students commonly disregarding the effects of the $f_{R}+f_{S}$ term on the output signal.

\section{E. (Case 5) Amplitude modulation present in the input signal}

Amplitude modulation in the form of a sinusoidal time dependent variance in the amplitude of the input signal is used in this tutorial to examine the effects of a time dependent amplitude on the output of the lock-in. Additionally, this case serves to demonstrate how the value of the time constant can affect the time required to take an accurate measurement. Since the output signal changes more slowly as the time constant increases, the time required for a stable output to be achieved in the most common case (case 1) will also increase as the time constant increases. This is important for students making use of the lock-in to make a large number of measurements to ensure that they select a time constant that is high enough to filter out any time varying components yet low enough to allow quick measurements to be taken. The average score on all pretest questions that demonstrate this case is slightly over $25 \%$. This weak performance demonstrates the student's limited understanding of the low-pass filter as well as how a change in the input signal's amplitude would affect the output signal. Students showed a marked improvement in performance after working on the tutorial with the average score being over $80 \%$ on post-test questions demonstrating this case.

\section{F. (Case 6) Two frequencies present in the input signal}

The final case involves the presence of two frequencies in the input signal with the $f_{R}-f_{S}$ component of each signal passing through the low-pass filter not strongly attenuated while both $f_{R}+f_{S}$ signals are strongly attenuated. This case serves to demonstrate to students how the lock-in processes multiple frequencies that are present in the input signal to generate a single output signal. It also acts as an example of the potential lab scenario in which the experimenter is attempting to measure the amplitude of one frequency (as in the most common case) but incorrectly sets the time constant too low, allowing part of the mixed signal from a deterministic interference with a frequency close to the value of the reference frequency to pass through the low-pass filter. The average student score on the pretest for all problems that demonstrate this case is $25 \%$. This low average score partly illustrates the students' inexperience with both cases involving two frequencies in the input signal and cases with frequencies in the input signal that do not equal the reference signal (similar to case 2). After students work on the tutorial, they obtain an average score of roughly $80 \%$ across all post-test questions that demonstrate this case.

Examining the pretest and post-test results of the six cases covered in Table II suggests that the tutorial is effective at improving student understanding in a variety of situations ranging from the most commonly experienced lab setting to less commonly faced cases that illustrate situations that the student may potentially face while making use of this device under less idealized circumstances. Despite the wide variety of experience levels among students who took the pretest and post-test there was little correlation between experience level and pretest scores with highly experienced students only performing slightly better on case 1 problems and all students performing comparably on all other cases. In addition to being based on a variety of potential experimental setups, the pretest and post-test questions can also be divided into two categories based upon what form the answer takes. Questions either ask the student to sketch the output signal that should result from a given set of input parameters or ask the student to fill in the blanks about characteristics of the input signal based on the lock-in's settings and a provided output signal diagram [24].

Questions in which the student was asked to predict and sketch the output signal were included to examine the student's ability to check the output signal to ensure that the input parameters are as they predicted. If the students are able to correctly answer problems of this type, they should be able to confirm that the output signal is as they predicted when performing a measurement in the lab and if this prediction does not match their measurement they can determine that the input signal has characteristics that they did not take into account. This ability to predict the form of the output signal before making a measurement should 
TABLE III. Summary of the average scores, standard deviation and number of instances of predicting input and predicting output questions as well as the total average score on both the pretest and post-test.

\begin{tabular}{lccccrr}
\hline \hline & $\begin{array}{c}\text { Average pretest } \\
\text { score }\end{array}$ & $\begin{array}{c}\text { Pretest standard } \\
\text { deviation }\end{array}$ & $\begin{array}{c}\text { Number of } \\
\text { instances in } \\
\text { pretest }\end{array}$ & $\begin{array}{c}\text { Average post-test } \\
\text { score }\end{array}$ & $\begin{array}{c}\text { Posttest Standard } \\
\text { Deviation }\end{array}$ & $\begin{array}{c}\text { Number of } \\
\text { instances in } \\
\text { post-test }\end{array}$ \\
\hline Predicting output & $38.1 \%$ & $42.6 \%$ & 135 & $87.7 \%$ & $27.7 \%$ & 151 \\
Predicting input & $42.5 \%$ & $42.1 \%$ & 55 & $80.9 \%$ & $30.5 \%$ & $<0.001$ \\
Total score & $39.4 \%$ & $42.5 \%$ & 190 & $85.9 \%$ & $28.7 \%$ & $<0.001$ \\
\hline \hline
\end{tabular}

ensure that students are less likely to record anomalous outputs based on an incorrect understanding of the input signal that they are analyzing. Students initially showed a limited ability to predict the output signal for the array of cases that were treated in the pretest. Table III shows that this resulted in an average score of under $40 \%$ across all pretest questions that had the student sketch the output signal. This average improves to over $85 \%$ after students made use of the tutorial.

The remaining questions, which ask students to predict the characteristics of the input signal based on the output signal and the lock-in's settings, evaluate the students' ability to examine an output signal and determine what the input signal must be. This ability is valuable to students in the lab setting when they are faced with an output signal that differs from what they expected. Table III shows that student performance on these questions before exposure to the tutorial is roughly $40 \%$ across all pretest questions that asked the student to predict the input settings. This average improved to over $80 \%$ on all post-test material as shown in Table III. Improved student performance on either of these two types of problems should correlate with increased student ability to troubleshoot difficulties that may arise when making use of the lock-in in their authentic research scenarios.

\section{SUMMARY}

We have developed and evaluated a research-based tutorial that helps students learn the basics of how this instrument operates and also helps students make connections between the relevant concepts and the pertinent mathematics that describes the operations of its major components. This tutorial helps students predict output signals and input signals in a variety of cases all of which mimic situations that may be faced by the student in the lab setting.

We find that physics graduate students who use lock-ins for their experimental research have many common difficulties with the basic functions of this instrument. These difficulties make it difficult for students to correctly make predictions about the output signal given the input parameters or input parameters given the output signal in any but the most common case with any degree of accuracy. Interviews suggest that the understanding of the majority of students is usually limited to the use of the lock-in as a black box to perform measurements which makes it extremely difficult for them to be able to troubleshoot problems (sometimes due to spurious signals that they may not be aware of or choosing incorrect time constant).

Examination of the average student scores on the pretests and post-tests shows considerable improvement for all cases discussed. This improved ability to identify both typical (case 1) and less common (cases 2-6) characteristics should improve student ability to both ensure that the output signals obtained are as expected and troubleshoot anomalous output signals or unexpected conditions present in the input signal when they arise. Additionally, this increase in score on the post-test across a wide variety of students with varied levels of experience with the lock-in demonstrates the tutorial's utility in both providing an "on ramp" for students who are just being introduced into the lab setting as well as providing an opportunity for those who have been using the lock-in to improve their understanding of this powerful device.

\section{ACKNOWLEDGMENTS}

We thank the National Science Foundation for Grant No. NSF-1124131.
[1] P. A. Temple, An introduction to phase-sensitive amplifiers: An inexpensive student instrument, Am. J. Phys. 43, 801 (1975).

[2] R. Wolfson, The lock-in amplifier: A student experiment, Am. J. Phys. 59, 569 (1991).
[3] J. H. Scofield, Frequency-domain description of a lock-in amplifier, Am. J. Phys. 62, 129 (1994).

[4] E. Marín and R. Ivanov, LIA in a Nut Shell: How can Trigonometry help to understand Lock-in Amplifier operation?, Latin-Am. J. Phys. Educ. 3, 544 (2009). 
[5] K. Edmondson, S. Agoston, and R. Ranganathan, Impurity level lifetime measurements using a lock-in amplifier, Am. J. Phys. 64, 787 (1996).

[6] S. DeVore, A. Gauthier, J. Levy, and C. Singh, Improving students' understanding of Lock-in amplifiers, Proceedings of the 2013 Physics Education Research Conference, Portland, OR, 2014, edited by P. V. Engelhardt, A. D. Churukian, and D. L. Jones, pp. 121-124 [arXiv:1601.00731].

[7] C. E. Wieman, W. K. Adams, and K. K. Perkins, PhET: Simulations that enhance learning, Science 322, 682 (2008).

[8] C. Crouch and E. Mazur, Peer Instruction: Ten years of experience, and results. Am. J. Phys. 69, 970 (2001).

[9] M. Windelspecht, Technology in the Freshman Biology Classroom: Breaking the Dual Learning Curve, Am. Biol. Teach. 63, 96 (2001).

[10] F. Reif and L. A. Scott, Teaching scientific thinking skills: Students, and computers coaching each other, Am. J. Phys. 67, 819 (1999).

[11] L. Hsu and K. Heller, Computer Problem Solving coaches, Proceedings of the National Association for the Advancement of Science Teaching (NARST 2009). Available http://groups .physics.umn.edu/physed/Talks/Hsu_NARST09_paper.pdf.

[12] C. Y. Chang, A problem-solving based computer-assisted tutorial for the earth sciences, J. Comput. Assist. Learn. 17, 263 (2001).

[13] S. Yalçinalp, Ö. Geban, and I. Özkan, Effectiveness of Using Computer-Assisted Supplementary Instruction for Teaching the Mole Concept, J. Res. Sci. Teach. 32, 1083 (2006); C. Singh, Interactive Video Tutorials for Enhancing Problem-Solving, Reasoning, and Meta-Cognitive Skills of Introductory Physics Students, AIP Conf. Proc. 720, 177 (2004); Problem Solving and Learning, AIP Conf. Proc. 1140, 183 (2009).

[14] A. Korkmaz and W. S. Harwood, Web-Supported Chemistry Education: Design of an Online Tutorial for Learning Molecular Symmetry, J. Sci. Educ. Technol. 13, 243 (2004).
[15] G. MacKinnon and P. Williams, Models for Integrating Technology in Higher Education: The Physics of Sound, J. Coll. Sci. Teach. 35, 22 (2006).

[16] K. C. Chu, The development of a web-based teaching system for engineering education, Engineering Science and Education Journal 8, 115 (1999).

[17] G. A. Sowell and R. Fuller, Some "Do's" and "Don't's" for Using Computers in Science Instruction, J. Coll. Sci. Teach. 20, 90 (1990).

[18] C.-L. C. Kulik and J. A. Kulik, Effectiveness of computerbased instruction: An updated analysis, Computers in Human Behavior, Computers Human Behav. 7, 75 (1991).

[19] G. MacKinnon, Computers in the science teacher education, J. Coll. Sci. Teach. 27, 305 (1998).

[20] H. Hemming, D. A. Day, and G. MacKinnon, Teaching in the age of technology: Tensions, and possibilities, Int. J. Techn. Know. Soc. 3, 63 (2007).

[21] S. DeVore, A. Gauthier, J. Levy, and C. Singh, Improving student understanding of Lock-in amplifiers, Am. J. Phys. 84, 52 (2016).

[22] D. G. Cahill, Analysis of heat flow in layered structures for time-domain thermoreflectance, Rev. Sci. Instrum. 75, 5119 (2004).

[23] F. Kremer and A. Schönhals, Broadband Dielectric Spectroscopy (Springer-Verlag Berlin Heidelberg, New York, 2003).

[24] The final version of the tutorial and supplementary material are available for download on ComPADRE at http://www .compadre.org/portal/items/detail.cfm?ID=13360. The simulation requires the installation of the LabVIEW Run Time Engine for use (a link to download this engine is available within the tutorial).

[25] L. McDermott, Peter Shaffer and the Physics Education group at the University of Washington, Tutorials in Introductory Physics, (Prentice Hall, Englewood Cliffs, NJ, 1988). 